\title{
Testing capability indices for one-sided processes with measurement errors
}

\author{
D. $\mathrm{Grau}^{\star}$
}

Laboratory of Applied Mathematics, CNRS UMR 5142 IUT de Bayonne, Université de Pau et des Pays de l'Adour, 17 place Paul Bert, 64100 Bayonne, France

Received: 4 September 2012 / Accepted: 10 July 2013

\begin{abstract}
In the manufacturing industry, many product characteristics are of one-sided tolerances. The process capability indices $C_{p}^{u}(u, v)$ and $C_{p}^{l}(u, v)$ can be used to measure process performance. Most research work related to capability indices assumes no gauge measurement errors. This assumption insufficiently reflects real situations even when advanced measuring instruments are used. In this paper we show that using a critical value without taking into account these errors, severely underestimates the $\alpha$-risk which causes a less accurate testing capacity. In order to improve the results we suggest the use of an adjusted critical value, and we give a Maple program to get it. An example in a polymer granulates manufactory is presented to illustrate this approach.
\end{abstract}

Keywords: Process capability indices; gauge measurement error; one-sided tolerance process; critical value; power test

\section{Introduction}

A common way to summarize the process performance is by using the process capability indices which provide information with respect to engineering specification. The two capability indices which have been widely used in manufacturing industry are $C_{p}$ and $C_{p k}$. The $C_{p}$ index measures only the potential of a process to produce acceptable product, and does not take into account where the process is centred. $C_{p k}$ index is computed using both location and dispersion information about the process. However, the indices $C_{p}$ and $C_{p k}$ consider only the process mean and standard deviation, but not the proximity of the process mean to the target value of the process characteristic. Chan et al. [1] proposed the $C_{p m}$ index to consider the proximity of the process mean to the target value. The $C_{p m k}$ index suggested by Pearn et al. [2] is a combination of $C_{p k}$ and $C_{p m}$, so it has the advantages of both $C_{p k}$ and $C_{p m}$. For a process with lower and upper tolerance limits $L S L$ and $U S L$, and a target $T$ set to the midpoint $m=(L S L+U S L) / 2$ of the tolerance interval, Vännman [3] constructed a unified superstructure for the four previous basic indices which can be defined as

$$
C_{p}(u, v)=\frac{d-u|\mu-m|}{3 \sqrt{\sigma^{2}+v(\mu-T)^{2}}},
$$

where $d=(U S L-L S L) / 2$ is the half-length of the tolerance interval, $\mu$ is the mean, $\sigma$ is the standard deviation, and $u$ and $v$ are two non-negative parameters. Although

\footnotetext{
* Correspondence: daniel.grau@univ-pau.fr
}

cases with symmetric tolerances $(T=m)$ are common in practical situations, cases with asymmetric tolerances $(T \neq m)$ often occur in manufacturing industry. In general asymmetric tolerances reflect that deviations from the target are less tolerable in one direction than in the other. In this situation Chen and Pearn [4] suggested to use the family

$$
C_{p}^{\prime \prime}(u, v)=\frac{d^{*}-u A^{*}}{3 \sqrt{\sigma^{2}+v A^{2}}},
$$

where $A=\max \left\{d(\mu-T) / D_{u}, d(T-\mu) / D_{l}\right\}, A^{*}=$ $\max \left\{d^{*}(\mu-T) / D_{u}, d^{*}(T-\mu) / D_{l}\right\}, D_{u}=U S L-T$, $D_{l}=T-L S L$, and $d^{*}=\min \left\{D_{u}, D_{l}\right\}$. Grau [5] suggests choosing parameters $u$ and $v$ according to the importance the user attaches to the process centring and/or to the percentage of non-conforming, which are the most important criteria to measure the process performance.

This paper considers the situation where a shift of the mean from the target in one direction appears less serious to the user, so that he is induced to define only one tolerance. Grau [6] suggests to the user to quantify the risk he considers the least serious when the mean shifts in the opposite direction from the target. When the risk is considered as $k$ times less serious, Grau [6] suggests using the families

$$
C_{p}^{u}(u, v)=\frac{D_{u}-u A_{u}^{*}}{3 \sqrt{\sigma^{2}+v A_{u}^{*^{2}}}}, \text { and } C_{p}^{l}(u, v)=\frac{D_{l}-u A_{l}^{*}}{3 \sqrt{\sigma^{2}+v A_{l}^{*^{2}}}},
$$

for an upper and a lower tolerance, where $A_{u}^{*}=\max (\mu-$ $T,(T-\mu) / k)$, and $A_{l}^{*}=\max \left((\mu-T) / k, T^{*}-\mu\right)$. Note that the letter $u$ used in superscript is an abbreviation of "upper", and so too is independent of the first of the 
two parameters $(u, v)$ of the indices family. The choice of the constant $k(\geqslant 1)$ is approximate, thus $C_{p}^{u}(u, v)$ and $C_{p}^{l}(u, v)$ have been constructed so that they are independent of $k$ when the mean shifts toward the tolerance limit. Note that the choice $k=1$ comes to take into account similar risks on the right and on the left. If for instance the risk on the left is considered as nil, taking $k$ infinite is sufficient. In this case when $\mu<T, C_{p}^{u}(u, v)=D_{u} /(3 \sigma)$, which is independent of $\mu$, and which is nothing but the potential capability $C_{p}$. For $C_{p}^{u}(u, v)$ and $C_{p}^{l}(u, v)$ Grau [7] suggests choosing parameters $u$ and $v$ according to the importance the user attaches to the process centring and/or to the percentage of non-conforming.

The capability indices calculated from data obtained from samples of manufactured items are not the true values but only estimations. Therefore, a certain amount of uncertainty, due to the sampling error, is necessarily present in the evaluation of the process performance, and in order to avoid erroneous interpretations, it is important to know the statistical properties of the estimators. A further source of uncertainty is given by the measurements errors. Mittag [8] was the first author to link capability indices and measurement errors. For the $C_{p}, C_{p k}, C_{P U}, C_{P L}$, $C_{p m}, C_{p m k}$ and $C_{p}^{\prime \prime}(u, v)$ indices, Pearn and Liao [9-11], Pearn et al. [12], Hsu et al. [13], Grau [14, 15] establish reliable lower confidence bounds and reliable critical values to estimate and test the process capability with gauge measurements errors.

In this paper, we consider the one-sided process capability indices $C_{p}^{u}(u, v)$ and $C_{p}^{l}(u, v)$, and construct a critical value to test whether a process is capable when gauge measurement errors are present.

This article is organized as follows. In Section 2, for one-sided tolerance process we quickly report the main results concerning the effects of gauge measurement errors on theoretical capability indices $C_{p}^{u}(u, v)$ and $C_{p}^{l}(u, v)$. In Section 3 we give the sampling distribution of capability indices in the presence of measurements errors. Sections 4 and 5 give critical values and adjusted critical values to test process capability when gauge measurement errors are noticed. Finally, a real example is presented in the last section.

\section{The indices $C_{p}^{u}(u, v), C_{p}^{\prime}(u, v)$, and the gauge measurement errors}

Suppose that the gauge measurement errors can be described as a random variable $M \sim N\left(0, \sigma_{M}^{2}\right)$. Mittag [8] define the degree of error contamination as

$$
\tau=\frac{\sigma_{M}}{\sigma} .
$$

Suppose that $X \sim N\left(\mu, \sigma^{2}\right)$ represents the relevant quality characteristic of the manufacturing process. In practice the observed variable $G$ (with gauge measurement errors) is measured rather than the true variable $X$. It is further assumed that $X$ and $M$ are additively linked according to $G=X+M$ and that $X$ and $M$ are stochastically independent. Then we have $G \sim N\left(\mu, \sigma_{G}^{2}=\sigma^{2}+\sigma_{M}^{2}\right)$ and the empirical process capability indices $C_{p}^{u G}(u, v)$ and $C_{p}^{l G}(u, v)$ are obtained by formula (1) after substituting $\sigma_{G}$ for $\sigma$.

$$
\begin{aligned}
C_{p}^{u G}(u, v) & =\frac{D_{u}-u A_{u}^{*}}{3 \sqrt{\sigma_{G}^{2}+v A_{u}^{*^{2}}}} \\
C_{p}^{l G}(u, v) & =\frac{D_{l}-u A_{l}^{*}}{3 \sqrt{\sigma_{G}^{2}+v A_{l}^{*^{2}}}} .
\end{aligned}
$$

Obviously if $\sigma_{M}=0$, then the empirical process capability, $C_{p}^{u G}(u, v)$ and $C_{p}^{l G}(u, v)$, reduce to the basic indices, $C_{p}^{u}(u, v)$ and $C_{p}^{l}(u, v)$. Since the variation of the observed data is greater than the variation of the original data, the denominator of the indices $C_{p}^{u G}(u, v)$ and $C_{p}^{l G}(u, v)$ becomes larger and we would understate the true capability of the process if the empirical data are used. The relation between $C_{p}^{u}(u, v), C_{p}^{l}(u, v)$, the true process capability, and $C_{p}^{u G}(u, v), C_{p}^{l G}(u, v)$, the empirical process capability, can be expressed as

$$
\begin{aligned}
& C_{p}^{u G}(u, v)=\frac{\sqrt{1+v \xi^{u^{2}}}}{\sqrt{1+\tau^{2}+v \xi^{u^{2}}}} C_{p}^{u}(u, v), \\
& C_{p}^{l G}(u, v)=\frac{\sqrt{1+v \xi^{l^{2}}}}{\sqrt{1+\tau^{2}+v \xi^{l^{2}}}} C_{p}^{l}(u, v)
\end{aligned}
$$

where $\xi^{u}=\max (\xi,-\xi / k), \xi^{l}=\max (\xi / k,-\xi)$ and $\xi=$ $(\mu-T) / \sigma$. It is clear that the ratio $C_{p}^{u G}(u, v) / C_{p}^{u}(u, v)$ is decreasing function of $\tau$. Then the measurements errors understate the true theoretical capability of the process. However, the theoretical capability is unknown and is estimated from sample data. Thus, in the following sections we deal with the effects of measurement errors on the behavior of estimated capability indices.

\section{Sampling distribution of $\hat{C}_{p}^{u G}(u, v)$ and $\hat{C}_{p}^{I G}(u, v)$}

Subsequently we will only develop the case of an upper tolerance and without difficulty will deduce the similar results in the case of a lower tolerance.

Let $X_{1}, X_{2}, \ldots, X_{n}$ be a random sample from a normal distribution with mean $\mu$ and variance $\sigma^{2}$ measuring the characteristic of the process. To estimate the $C_{p}^{u}(u, v)$ and $C_{p}^{l}(u, v)$ indices, we replace $\mu$ and $\sigma^{2}$ by their conventional estimators $\bar{X}=\left(\sum_{i=1}^{n} X_{i}\right) / n$, and $S^{2}=\sum_{i=1}^{n}\left(X_{i}-\bar{X}\right)^{2} / n$. Thus we define the estimators $\hat{C}_{p}^{u}(u, v)$ and $\hat{C}_{p}^{l}(u, v)$ as

$$
\begin{aligned}
& \hat{C}_{p}^{u}(u, v)=\frac{D_{u}-u \hat{A}_{u}^{*}}{3 \sqrt{S^{2}+v \hat{A}_{u}^{*^{2}}}}, \\
& \hat{C}_{p}^{l}(u, v)=\frac{D_{l}-u \hat{A}_{l}^{*}}{3 \sqrt{S^{2}+v \hat{A}_{l}^{*^{2}}}},
\end{aligned}
$$




$$
\xi^{u}=\frac{-C_{p}^{u} u / 3+\sqrt{\left(C_{p}^{u}\right)^{2}(u / 3)^{2}+\left(\left(C_{p}^{u}\right)^{2}-c^{2}\right)\left(v c^{2}-(u / 3)^{2}\right)}}{v c^{2}-(u / 3)^{2}} .
$$

where $\hat{A}_{u}^{*}=\max (\bar{X}-T,(T-\bar{X}) / k)$ and $\hat{A}_{l}^{*}=$ $\max ((\bar{X}-T) / k, T-\bar{X})$.

Under the assumption of normality, and for $(u, v) \neq$ $(0,0)$, Grau [7] gives the cumulative distribution function of $\hat{C}_{p}^{u}(u, v)$ which can be rewritten as

$$
F_{\hat{C}_{p}^{u}(u, v)}(x)=1-\int_{0}^{K(x)} H(x, t) d t, \text { for } x>0,
$$

where $K(x)=B_{u} \sqrt{n} /(u+3 x \sqrt{v}), B_{u}=D_{u} / \sigma=3 C_{p}^{u}$ with $C_{p}^{u}=C_{p}^{u}(0,0)$,

$H(x, t)=F_{K}\left(\left(\frac{B_{u} \sqrt{n}-u t}{3 x}\right)^{2}-v t^{2}\right) f_{Z_{u}}(t)$, with $F_{K}(x)$ is the cumulative $\chi_{n-1}^{2}$ distribution, $f_{Z_{u}}(t)=\phi(t-\sqrt{n} \xi)+$ $k \phi(k t+\sqrt{n} \xi)$ and $\phi(x)$ is the probability density of the standard normal distribution $N(0,1)$.

For $C_{p}^{l}(u, v)$, we obtain similar results replacing $B_{u}$ by $B_{l}=D_{l} / \sigma=3 C_{p}^{l}$ with $C_{p}^{l}=C_{p}^{l}(0,0)$, and $f_{Z_{u}}(t)$ by $f_{Z_{l}}(t)=k \phi(k t-\sqrt{n} \xi)+\phi(t+\sqrt{n} \xi)$.

When there are gauge measurement errors, the variable $G$ is measured rather than the true variable $X$. $\left(G_{1}, G_{2}, \ldots, G_{n}\right)$ are selected randomly from a stable process following a normal distribution $N\left(\mu, \sigma_{G}^{2}\right)$. We denote, $\bar{G}=\sum_{i=1}^{n} G_{i} / n, S_{G}^{2}=\sum_{i=1}^{n}\left(G_{i}-\bar{G}\right)^{2} / n, \xi_{G}^{u}=$ $\max \left(\xi_{G},-\xi_{G} / k\right), \xi_{G}^{l}=\max \left(\xi_{G} / k,-\xi_{G}\right)$ and $\xi_{G}=(\mu-$ $T) / \sigma_{G}$, and consider the following natural estimators $\hat{C}_{p}^{u G}(u, v)$ and $\hat{C}_{p}^{l G}(u, v)$ defined as

$$
\begin{aligned}
\hat{C}_{p}^{u G}(u, v) & =\frac{D_{u}-u \hat{A}_{u}^{* G}}{3 \sqrt{S_{G}^{2}+v \hat{A}_{u}^{* G^{2}}}}, \\
\hat{C}_{p}^{l G}(u, v) & =\frac{D_{l}-u \hat{A}_{l}^{* G}}{3 \sqrt{S_{G}^{2}+v \hat{A}_{l}^{* G^{2}}}},
\end{aligned}
$$

where $\hat{A}_{u}^{* G}=\max (\bar{G}-T,(T-\bar{G}) / k)$ and $\hat{A}_{l}^{* G}=$ $\max ((\bar{G}-T) / k, T-\bar{G})$.

Thus for $(u, v) \neq(0,0)$, the cumulative distribution of $\hat{C}_{p}^{u G}(u, v)$ is

$$
F_{\hat{C}_{p}^{u G}(u, v)}(x)=1-\int_{0}^{K_{G}(x)} H_{G}(x, t) d t, \text { for } x>0,
$$

where $K_{G}(x)=B_{u}^{G} \sqrt{n} /(u+3 x \sqrt{v}), B_{u}^{G}=D_{u} / \sigma_{G}=$ $3 C_{p}^{u G}$ with $C_{p}^{u G}=C_{p}^{u G}(0,0)$,

$$
\begin{aligned}
H_{G}(x, t) & =F_{K}\left(\left(\frac{B_{u}^{G} \sqrt{n}-u t}{3 x}\right)^{2}-v t^{2}\right) f_{Z_{u}^{G}}(t), \\
\text { with } f_{Z_{u}^{G}}(t) & =\phi\left(t-\sqrt{n} \xi_{G}\right)+k \phi\left(k t+\sqrt{n} \xi_{G}\right) .
\end{aligned}
$$

For $\hat{C}_{p}^{l G}(u, v)$, we obtain similar results replacing $B_{u}^{G}$ by $B_{l}^{G}=D_{l} / \sigma_{G}=3 C_{p}^{l G}$ with $C_{p}^{l G}=C_{p}^{l G}(0,0)$, and $f_{Z_{u}^{G}}(t)$ by $f_{Z_{l}^{G}}(t)=k \phi\left(k t-\sqrt{n} \xi_{G}\right)+\phi\left(t+\sqrt{n} \xi_{G}\right)$.

\section{Capability testing based on $\hat{C}_{p}^{u G}(u, v)$ or $\hat{C}_{p}^{I G}(u, v)$}

To determine whether a given process meets the preset capability requirement, we could consider the following statistical hypotheses testing:

- $H_{0}: C_{p}^{u}(u, v) \leqslant c$ Process is not capable;

- $H_{1}: C_{p}^{u}(u, v)>c$ Process is capable;

where $c$ is the required process capability. If the calculated process capability $\hat{c}_{p}^{u}(u, v)$ is greater than the critical value $c_{0}$, we reject the null hypothesis and conclude that the process is capable with error $\alpha$, which is the chance of incorrectly concluding an incapable process as capable. Given values of $c$ and $\alpha$, from (4) the critical value $c_{0}$ can be determined by solving the equation $\alpha=$ $P\left(\hat{C}_{p}^{u}(u, v)>c_{0} \mid C_{p}^{u}(u, v)=c\right)=\int_{0}^{K\left(c_{0}\right)} H\left(c_{0}, t\right) d t$, where from (A.9) in Appendix A, $B_{u}=3 C_{p}^{u}=3 \sqrt{1+v \xi^{u^{2}}} c+$ $u \xi^{u}$. However, since the process parameters $\mu$ and $\sigma$ are unknown, parameters $\xi$ and therefore $\xi^{u}$ are unknown. We replace $\xi$ and $\xi^{u}$ by the observation of the natural estimators $\hat{\xi}=(\bar{X}-T) / S$ and $\hat{\xi}^{u}=\max (\hat{\xi},-\hat{\xi} / k)$. Note that we use the same symbols $\hat{\xi}$ and $\hat{\xi}^{u}$ for the estimators as well as the estimations.

In the presence of measurements errors, $C_{p}^{u G}(u, v)$ is measured rather than the true variable $C_{p}^{u}(u, v)$. Thus the $\alpha$-risk denoted by $\alpha_{G}$ is defined as $\alpha_{G}=$ $P\left(\hat{C}_{p}^{u G}(u, v)>c_{0} \mid C_{p}^{u}(u, v)=c\right)$. Earlier discussions indicate that we underestimate the real process capability using $\hat{C}_{p}^{u G}(u, v)$ instead of $\hat{C}_{p}^{u}(u, v)$. The probability of $\hat{C}_{p}^{u G}(u, v)$ being greater than $c_{0}$ will be less important than by using $\hat{C}_{p}^{u}(u, v)$. Thus $\alpha_{G}$, the $\alpha$-risk using $\hat{C}_{p}^{u G}(u, v)$ to estimate $C_{p}^{u}(u, v)$ is less than $\alpha$, using $\hat{C}_{p}^{u}(u, v)$ when estimating $C_{p}^{u}(u, v)$. To illustrate the performance of $\alpha_{G}$ versus $\tau$, we have considered the particular case $u=0.5$, $v=1.5, k=3, c=1$ and $1.5, \alpha=0.05$, and have plotted curves $\alpha_{G}$ for various $C_{p}^{u}$ values. The critical value $c_{0}$ is obtained from (4) where $\xi$ is defined from (A.2) and $\xi^{u}$ is defined from (A.14) as

$$
\text { see equation above. }
$$

Then from (5) we compute $\alpha_{G}=\int_{0}^{K_{G}\left(c_{0}\right)} H_{G}\left(c_{0}, t\right) d t$, where $C_{p}^{u G}$ and $\xi_{G}$ are obtained from (A.12) and (A.5). Figure 1 plots $\alpha_{G}$ versus $\tau \in[0,1]$ with $n=$ $30,50,70,100,150$ for $C_{p}^{u}=c(0.5)(c+1)$. Note that for $\tau=0, \alpha_{G}=\alpha$. In Figure $1, \alpha_{G}$ decreases as $\tau$ or $n$ increases, and the decreasing rate is more significant with large $c$ and small $C_{p}^{u}$.

We now consider the power of the test, the chance of correctly concluding a capable process as capable. It can 


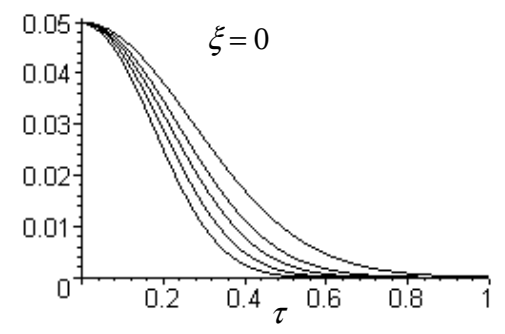

(a)
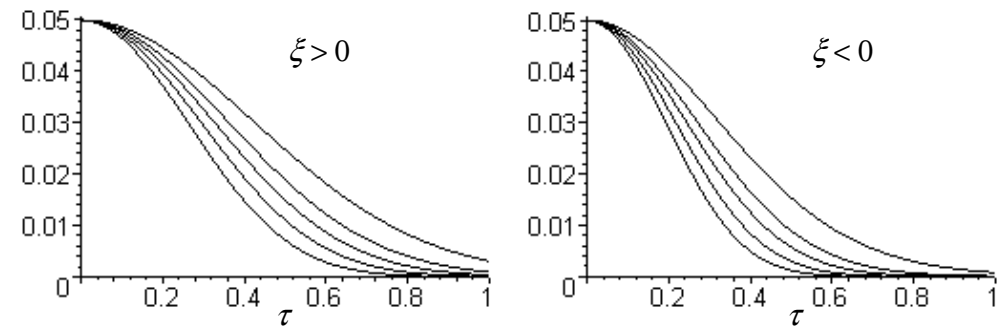

(b)
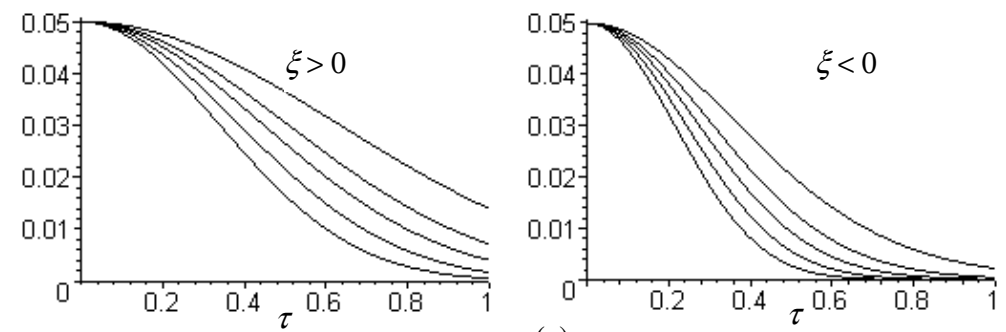

(c)
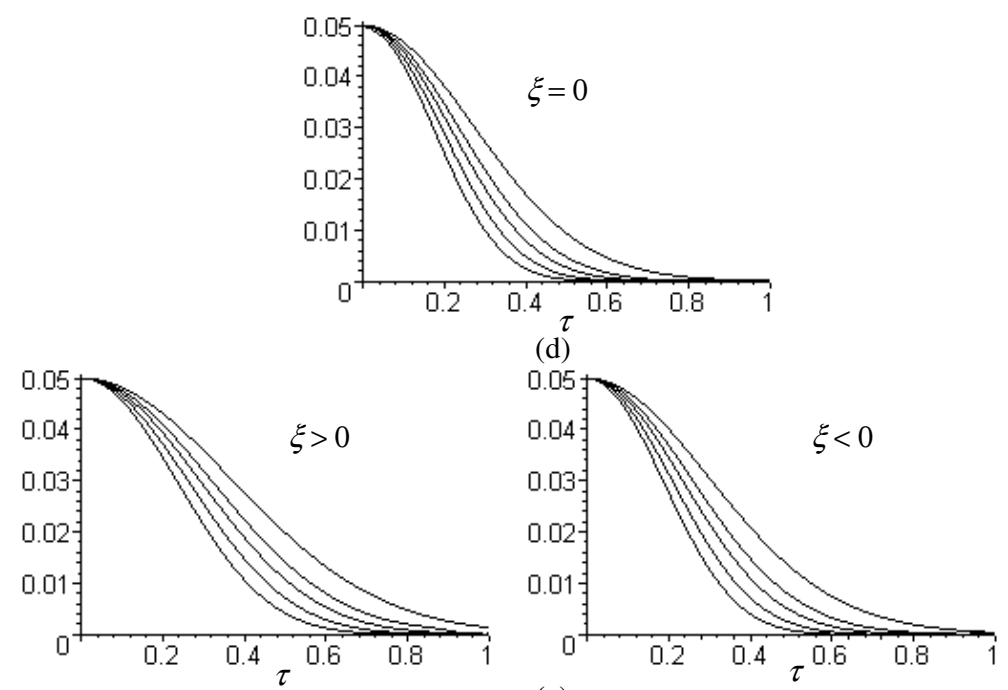

(e)
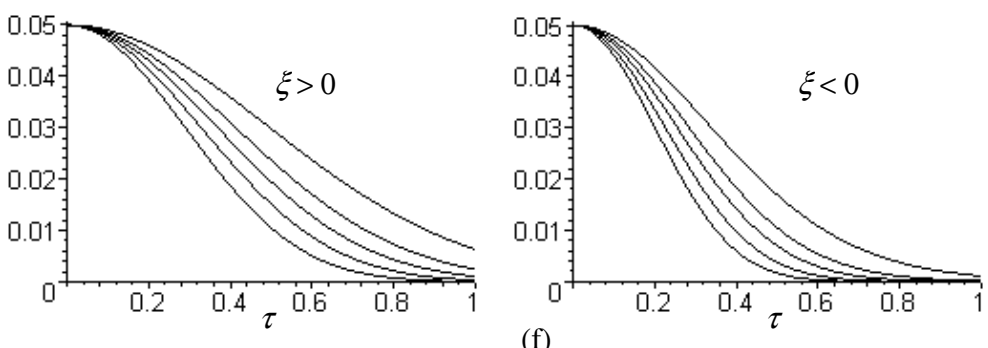

Fig. 1. Plots of $\alpha_{G}$ versus $\tau$ with $n=30,50,70,100,150$ (top to bottom), $u=0.5, v=1.5, k=3, \alpha=0.05$ for (a) $c=1$, $C_{p}^{u}=1$; (b) $c=1, C_{p}^{u}=1.5$; (c) $c=1, C_{p}^{u}=2$, (d) $c=1.5, C_{p}^{u}=1.5$; (e) $c=1.5, C_{p}^{u}=2$; (f) $c=1.5, C_{p}^{u}=2.5$. 
be computed as $\pi\left(C_{p}^{u}(u, v)\right)=P\left(\hat{C}_{p}^{u}(u, v)>c_{0} \mid C_{p}^{u}(u, v)\right)=$ $\int_{0}^{K\left(c_{0}\right)} H\left(c_{0}, t\right) d t$, with from (A.9) $B_{u}=3 C_{p}^{u}=$ $3 \sqrt{1+v \xi^{u^{2}}} c+u \xi^{u}$.

In the presence of measurements errors, the power of the test denoted by $\pi_{G}$ is as follows $\pi_{G}\left(C_{p}^{u}(u, v)\right)=$ $P\left(\hat{C}_{p}^{u G}(u, v)>c_{0} \mid C_{p}^{u}(u, v)\right)=\int_{0}^{K_{G}\left(c_{0}\right)} H_{G}\left(c_{0}, t\right) d t$. To illustrate the performance of $\pi_{G}$ versus $\tau$, we consider the particular case $u=0.5, v=1.5, k=3$ and plot the curves $\pi_{G}$ for various $C_{p}^{u}$ et $C_{p}^{u}(u, v)$ values. In the previous equation, $C_{p}^{u G}, \xi^{u}, \xi$ and $\xi_{G}$ are obtained from (A.12), (A.14), (A.2) and (A.5). Figure 2 plots $\pi_{G}$ versus $\tau \in[0,1]$ with $n=50, \alpha=0.05, c=1.00$ and $1.50, C_{p}^{u}(u, v)=$ $c(0.20)(c+1)$, and $C_{p}^{u}=C_{p}^{u}(u, v), C_{p}^{u}(u, v)+0.33$ and $C_{p}^{u}(u, v)+0.5$. In Figure 2, we see that $\pi_{G}$ decreases as $\tau$ increases and the decreasing rate is more significant with large $c$. The presence of measurements errors can have a very substantial effect on $\pi_{G}$. For instance, for $c=1.5$ and $C_{p}^{u}=C_{p}^{u}(u, v)=2.3$ (Fig. $2 \mathrm{~d}$ ), $\pi_{G}$ is approximately equal to 1 without measurement errors. However, when $\tau=1, \pi_{G}$ is approximately equal to 0.15 for $\xi>0$ and approximately equal to 0.41 for $\xi<0$.

\section{Adjusted critical values}

As we have seen in the previous section, the $\alpha$-risk and the test power decrease with measurement errors. The capability testing results would be misleading if the producers do not take account of the gauge measurements errors. Thus, in order to improve the test power, we revise the critical value, denoted by $c_{0}^{A}$, to satisfy $c_{0}^{A}<c_{0}$. Let $\alpha_{A}=P\left(\hat{C}_{p}^{u G}(u, v)>c_{0}^{A} \mid C_{p}^{u}(u, v)=c\right)$ and $\pi_{A}\left(C_{p}^{u}(u, v)\right)=P\left(\hat{C}_{p}^{u G}(u, v)>c_{0}^{A} \mid C_{p}^{u}(u, v)\right)$, be the $\alpha$-risk and the test power using the adjusted critical value. Since $c_{0}^{A}<c_{0}, P\left(\hat{C}_{p}^{u G}(u, v)>c_{0}^{A}\right)$ is greater than $P\left(\hat{C}_{p}^{u G}(u, v)>c_{0}\right)$, and both $\pi_{A}$ and $\alpha_{A}$ increase. To ensure that the $\alpha$-risk is within the preset magnitude, we set $\alpha_{A}=\alpha$, and solve equation $\alpha=P\left(\hat{C}_{p}^{u G}(u, v)>c_{0}^{A} \mid C_{p}^{u}(u, v)=c\right)$ to obtain $c_{0}^{A}$. Thus from (5), we must solve

$$
\alpha=\int_{0}^{K_{G}\left(c_{0}^{A}\right)} H_{G}\left(c_{0}^{A}, t\right) d t .
$$

Since the process parameters $\mu$ and $\sigma_{G}$ are unknown, then $\xi_{G}$ is also unknown. Thus the previous equation involves the unknown parameters $\xi_{G}$ and $C_{p}^{u G}$ or $C_{p}^{u}$ from (A.12). We replace $\xi_{G}$ by the observed value $\hat{\xi}_{G}$, and from (A.13) $C_{p}^{u}$ can be obtained by

$$
C_{p}^{u}=\sqrt{1+\tau^{2}}\left(\frac{\sqrt{1+v \hat{\xi}_{G}^{u^{2}}} \sqrt{1+v \hat{\xi}^{u^{2}}}}{\sqrt{1+\tau^{2}+v \hat{\xi}^{u^{2}}}} c+u \hat{\xi}_{G}^{u} / 3\right)
$$

where $\hat{\xi}_{G}^{u}$ and $\hat{\xi}^{u}$ are defined from (A.3) and (A.7) as follows

$$
\begin{aligned}
\hat{\xi}_{G}^{u} & =\max \left(\hat{\xi}_{G},-\hat{\xi}_{G} / k\right), \\
\text { and } \hat{\xi}^{u} & =\max \left(\hat{\xi}_{G} \sqrt{1+\tau^{2}},-\hat{\xi}_{G} \sqrt{1+\tau^{2}} / k\right) .
\end{aligned}
$$

With that adjusted critical value $c_{0}^{A}$, we can calculate the test power noted down as $\pi_{A}$. Let $C_{p 1}^{u}$ be the value of $C_{p}^{u}$ the solution of equation (7). The test power can be calculated as follows

$$
\begin{aligned}
\pi_{A}\left(C_{p}^{u}(u, v)\right) & =P\left(\hat{C}_{p}^{u G}(u, v)>c_{0}^{A} \mid C_{p}^{u}(u, v), C_{p}^{u}=C_{p 1}^{u}\right) \\
& =\int_{0}^{K_{1}\left(c_{0}^{A}\right)} H_{1}\left(c_{0}^{A}, t\right) d t
\end{aligned}
$$

with

$$
K_{1}(x)=B_{u 1}^{G} \sqrt{n} /(u+3 x \sqrt{v}),
$$

$B_{u 1}^{G}=3 C_{p}^{u G}=\frac{3 C_{p 1}^{u}}{\sqrt{1+\tau^{2}}}$ from (A.12),

$$
\begin{aligned}
H_{1}(x, t) & =F_{K}\left(\left(\frac{B_{u 1}^{G} \sqrt{n}-u t}{3 x}\right)^{2}-v t^{2}\right) f_{Z_{u 1}^{G}}(t), \\
f_{Z_{u 1}^{G}}(t) & =\phi\left(t-\sqrt{n} \xi_{G 1}\right)+k \phi\left(k t+\sqrt{n} \xi_{G 1}\right), \\
\xi_{G 1} & =\left\{\begin{array}{l}
\xi_{G 1}^{u} \text { if } \xi_{G 1}>0 \\
-k \xi_{G 1}^{u} \text { if } \xi_{G 1}<0
\end{array}\right. \text { from (A.4), } \\
\xi_{G 1}^{u} & =\xi_{1}^{u} / \sqrt{1+\tau^{2}} \text { from (A.8), }
\end{aligned}
$$

and $\xi_{1}^{u}$ is obtained substituting $C_{p 1}^{u}$ to $C_{p}^{u}$ in (A.14). $\xi_{G 1}$ and $\xi_{G}$ have the same sign, but $\xi_{G}$ is unknown. Thus we replace $\xi_{G}$ by the observed value $\hat{\xi}_{G}$, and replace $\xi_{G 1}$ by $\hat{\xi}_{G 1}$ defined by $\hat{\xi}_{G 1}=\left\{\begin{array}{l}\xi_{G 1}^{u} \text { if } \hat{\xi}_{G}>0 \\ -k \xi_{G 1}^{u} \text { if } \hat{\xi}_{G}<0\end{array}\right.$.

For a process with a lower tolerance, we obtain similar results replacing, $C_{p 1}^{u}$ by $C_{p 1}^{l}$ the solution of equation

$$
C_{p}^{l}=\sqrt{1+\tau^{2}}\left(\frac{\sqrt{1+v \hat{\xi}_{G}^{l^{2}}} \sqrt{1+v \hat{\xi}^{l^{2}}}}{\sqrt{1+\tau^{2}+v \hat{\xi}^{l^{2}}}} c+u \hat{\xi}_{G}^{l} / 3\right),
$$

$\hat{\xi}_{G}^{u} \quad$ by $\quad \hat{\xi}_{G}^{l}=\max \left(\hat{\xi}_{G} / k,-\hat{\xi}_{G}\right), \quad \hat{\xi}^{u}$ by $\quad \hat{\xi}^{l}=$ $\max \left(\hat{\xi}_{G} \sqrt{1+\tau^{2}} / k,-\hat{\xi}_{G} \sqrt{1+\tau^{2}}\right), \quad B_{u 1}^{G} \quad$ by $\quad B_{l 1}^{G}=$ $3 C_{p 1}^{l} / \sqrt{1+\tau^{2}}, \quad \hat{f}_{Z_{u 1}^{G}}(t)=\phi\left(t-\sqrt{n} \hat{\xi}_{G 1}\right)+$ $k \phi\left(k t+\sqrt{n} \hat{\xi}_{G 1}\right)$ by $\hat{f}_{Z_{l 1}^{G}}(t)=k \phi\left(k t-\sqrt{n} \hat{\xi}_{G 1}\right)+$ $\phi\left(t+\sqrt{n} \hat{\xi}_{G 1}\right)$, where $\hat{\xi}_{G 1}=\left\{\begin{array}{lll}k \xi_{G 1}^{l} & \text { if } & \hat{\xi}_{G}>0 \\ -\xi_{G 1}^{l} & \text { if } & \hat{\xi}_{G}<0\end{array}\right.$, $\xi_{G 1}^{l}=\xi_{1}^{l} / \sqrt{1+\tau^{2}}$, and $\xi_{1}^{l}$ is obtained substituting $C_{p 1}^{l}$ to $C_{p}^{l}$ in (A.14).

A Maple program is developed in Appendix B to compute the adjusted critical value $c_{0}^{A}$ and plot the power test. Figure 3 plots $\pi_{A}$ versus $\tau \in[0,1]$ with $u=0.5$, $v=1.5, k=3, n=50, \alpha=0.05, c=1.00,1.50$, 

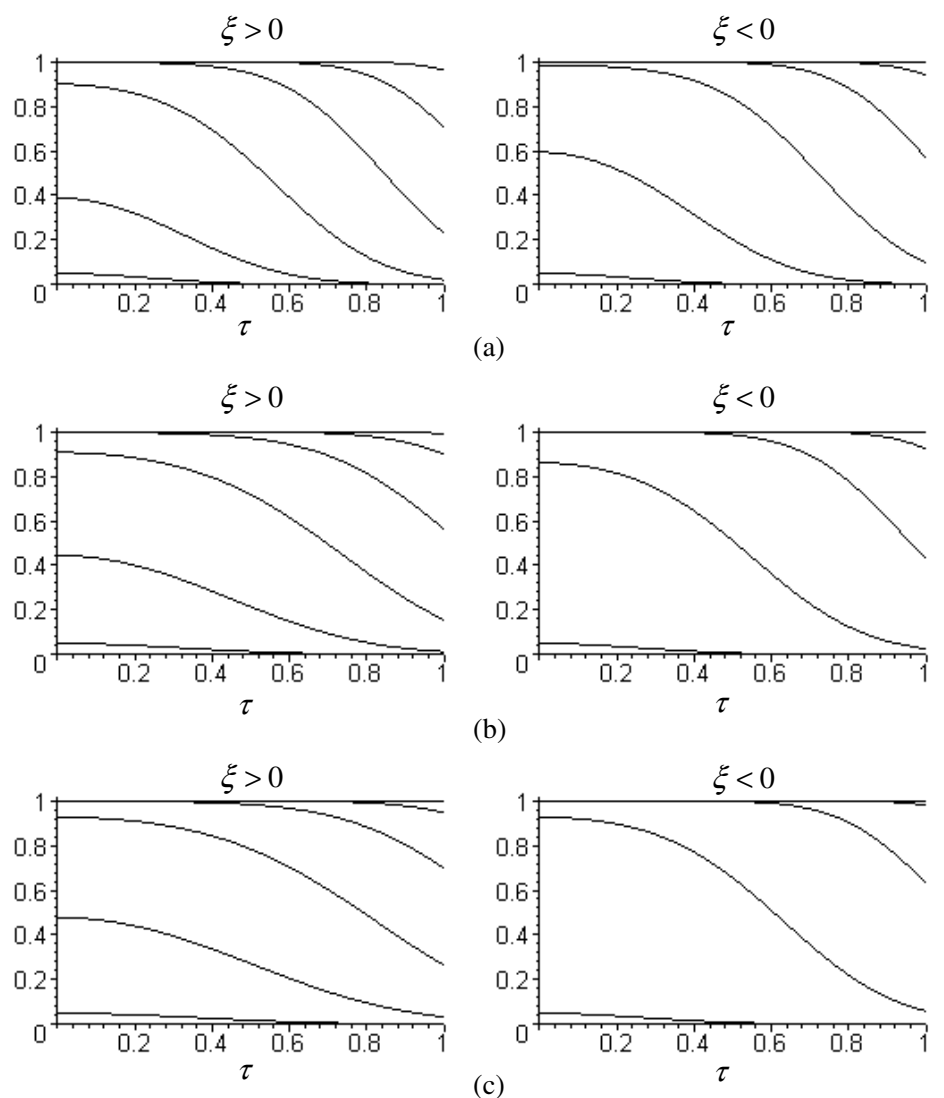

(b)
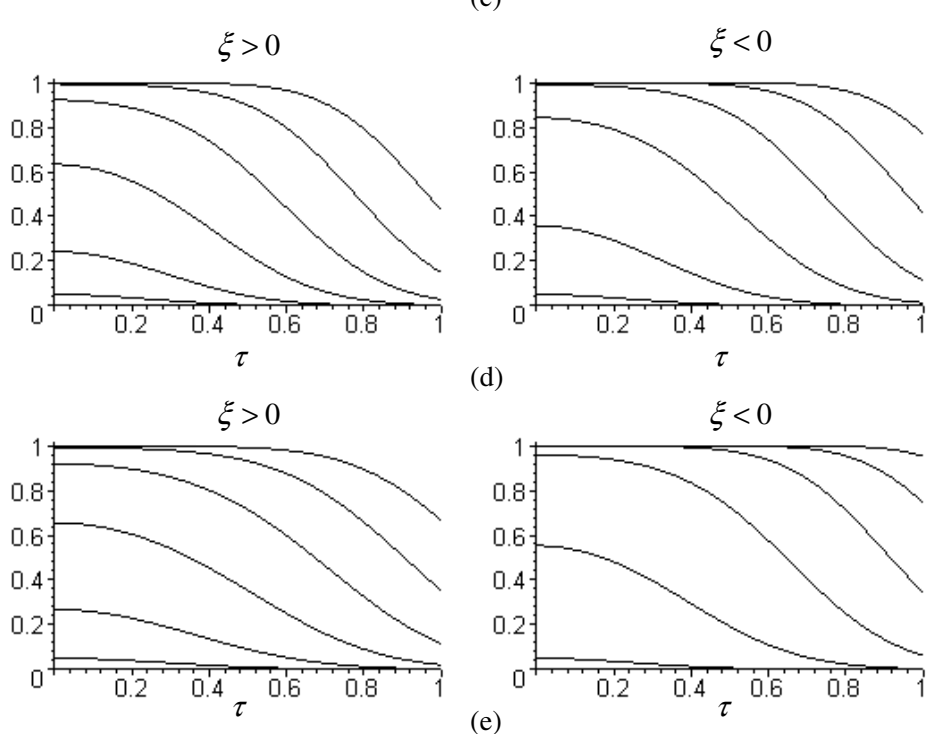

(d)
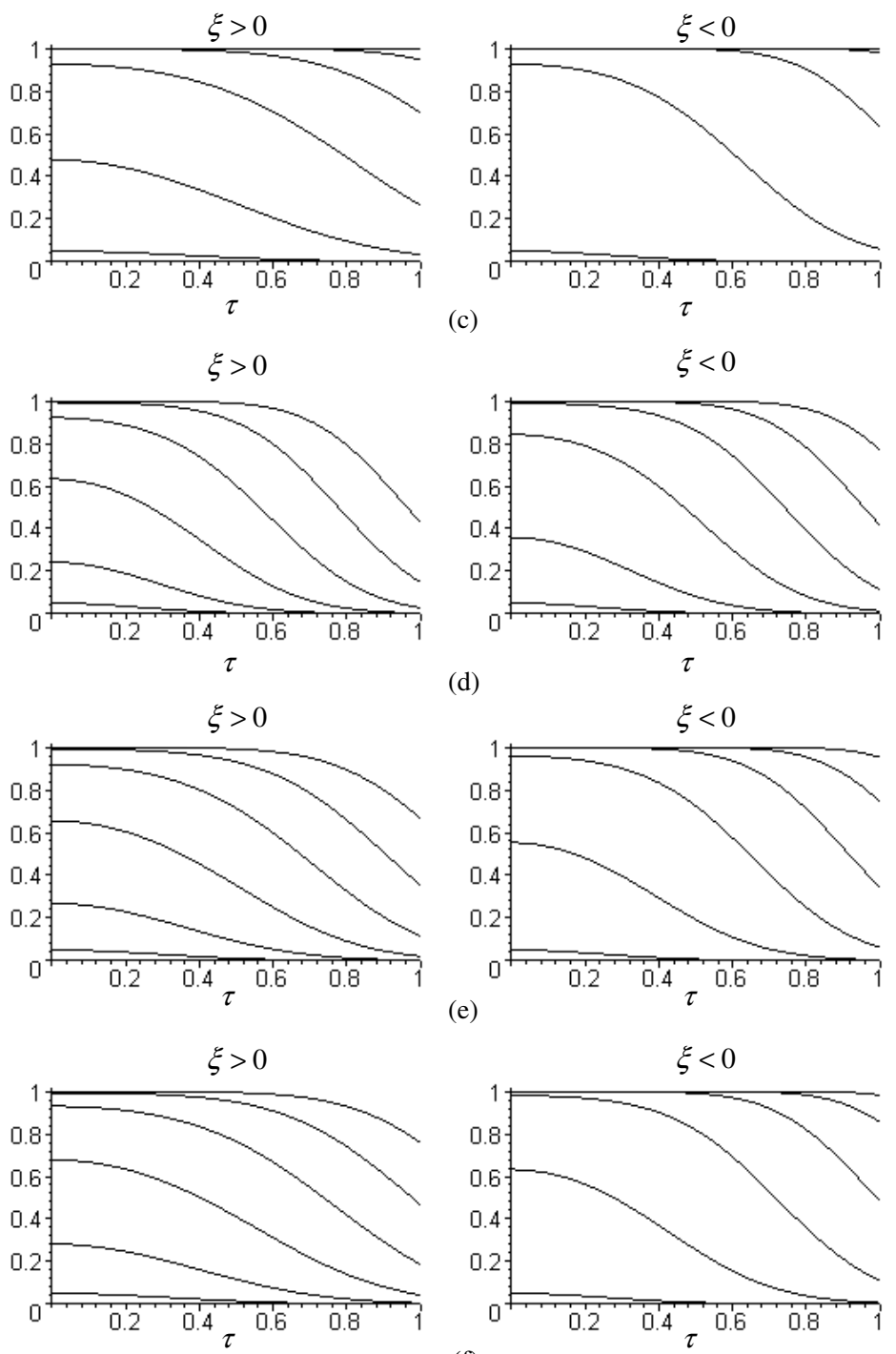

(f)

Fig. 2. Plots of $\pi_{G}$ versus $\tau$ with $u=0.5, v=1.5, k=3, n=50, \alpha=0.05, C_{p}^{u}(u, v)=c(0.20)(c+1)$ (bottom to top) for (a) $c=1$ and $C_{p}^{u}=C_{p}^{u}(u, v)$; (b) $c=1$ and $C_{p}^{u}=C_{p}^{u}(u, v)+0.33$; (c) $c=1$ and $C_{p}^{u}=C_{p}^{u}(u, v)+0.5$; (d) $c=1.5$ and $C_{p}^{u}=C_{p}^{u}(u, v)$; (e) $c=1.5$ and $C_{p}^{u}=C_{p}^{u}(u, v)+0.33 ;$ (f) $c=1.5$ and $C_{p}^{u}=C_{p}^{u}(u, v)+0.5$. 

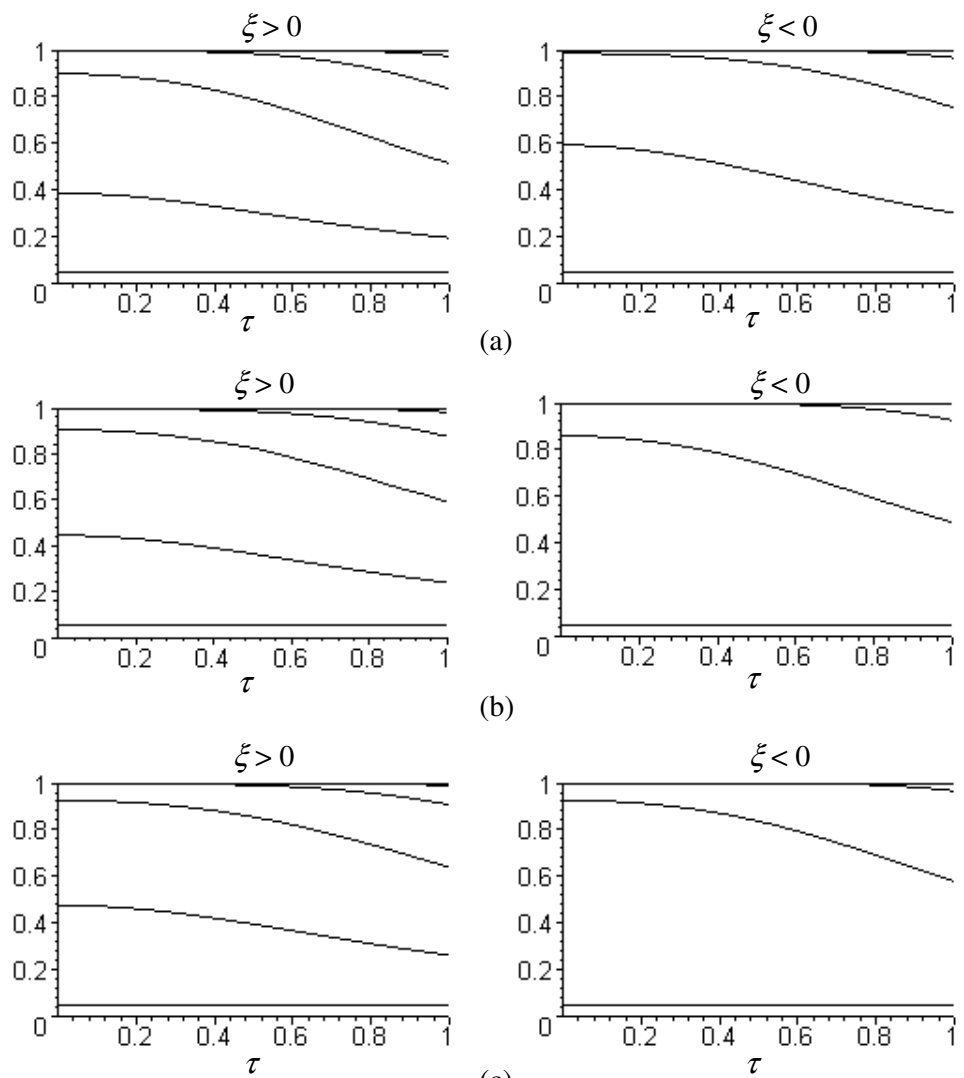

(c)
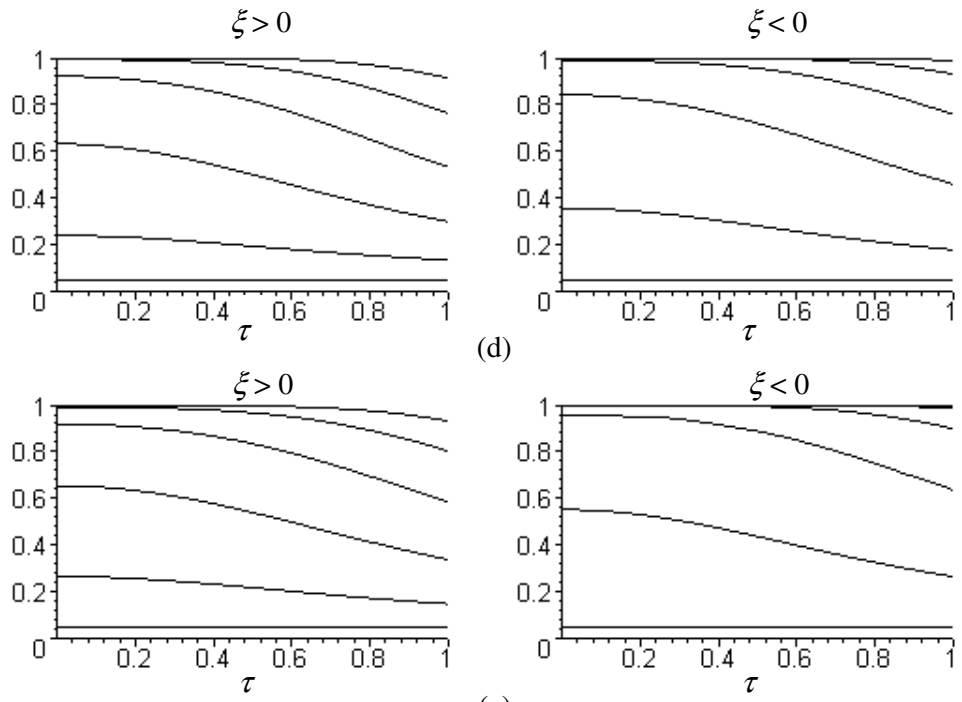

(e)
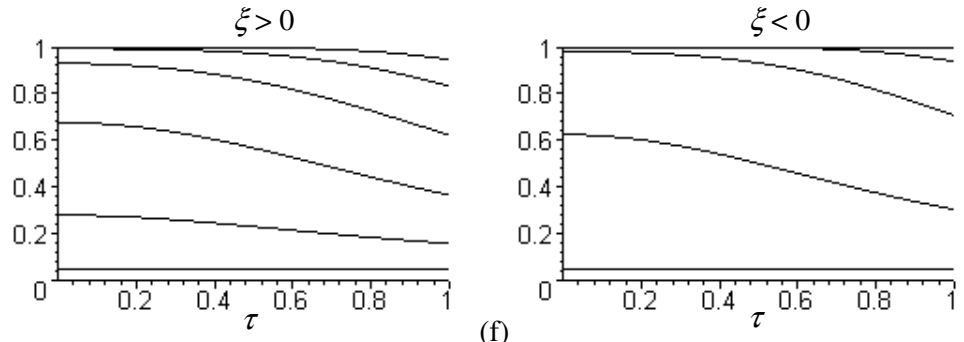

Fig. 3. Plots of $\pi_{A}$ versus $\tau$ with $u=0.5, v=1.5, k=3, n=50, \alpha=0.05, C_{p}^{u}(u, v)=c(0.20)(c+1)$ (bottom to top) for (a) $c=1$ and $C_{p}^{u}=C_{p}^{u}(u, v)$; (b) $c=1$ and $C_{p}^{u}=C_{p}^{u}(u, v)+0.33 ;(\mathrm{c}) c=1$ and $C_{p}^{u}=C_{p}^{u}(u, v)+0.5 ;(\mathrm{d}) c=1.5$ and $C_{p}^{u}=C_{p}^{u}(u, v)$; (e) $c=1.5$ and $C_{p}^{u}=C_{p}^{u}(u, v)+0.33$; (f) $c=1.5$ and $C_{p}^{u}=C_{p}^{u}(u, v)+0.5$. 
$C_{p}^{u}(u, v)=c(0.20)(c+1)$ and $C_{p}^{u}=C_{p}^{u}(u, v), C_{p}^{\prime \prime}(u, v)+$ 0.33 and $C_{p}^{u}(u, v)+0.5$. In Figure $3, \pi_{A}$ decreases as $\tau$ increases and the decreasing rate is more significant with large $c$. However, if we compare Figures 2 and 3, we can see that the powers corresponding to the adjusted critical values $c_{0}^{A}$ decrease more slowly. Thus the test power is improved.

\section{Example}

To illustrate our approach we go back to a study carried out within the Arkema company (Grau [7]). This company manufactures different sorts of polymer granulates which are transformed for many applications such as films or bottles used for food packaging. Polymer granulates are also used as tie layer in multilayer structures, providing outstanding adhesion between several materials, or coupling agent for reinforced thermoplastics. Before polymerization, an additive, maleic anhydride, is blended. For the studied grade, the target for this additive is $1500 \mathrm{ppm}$ and the necessary minimal value is $1000 \mathrm{ppm}$, i.e. the lower limit of tolerance. The cost of this additive not being very important, no upper limit has been fixed. During the manufacturing cycle, processes are considered capable when the capability indices are higher than 1.33. The objective of the Arkema company is to use a capability index with a clear interpretation in terms of centring and non-conforming. The selected index is $C_{p}^{l}(0.6,0.2)$. So a value of $C_{p}^{l}(0.6,0.2)$ superior or equal to 1.33 enables us to say that the process is capable as far as the percentage of manufactured non conforming items is inferior or equal to $50 \mathrm{ppm}$, and the process mean does not move away for more than $42 \%$ of the distance between the target and the lower tolerance limit (Grau [7]). We propose to determine whether the process is capable when the risk $k$ is equal to 3 , and the degree of error contamination $\tau$ is equal to 0.25 . A sample of 112 values of maleic anhydride was taken to estimate the capability of the manufacturing process. Ryan-Joiner test for normality was carried out pointing out that the sample can be regarded as taken from a normal distribution process. From the sample data we have $\bar{g}=1590.480, s_{G}=105.914$, $\hat{\xi}_{G}=0.854$, and $\hat{c}_{p}^{l G}(0.6,0.2)=1.505$, a value greater than 1.33. However this value is a specific point estimate that depends on the selected sample and does not make it possible to draw conclusions on the capability of the process. To decide whether the process is capable, that is to say if $C_{p}^{l}(0.6,0.2)>1.33$, we calculate the critical value using the method described in Section 5. Let the $\alpha$ risk be 0.05. From the Maple program in Appendix B, we obtain the critical value $c_{0}^{A}=1.456$. Since $\hat{c}_{p}^{l G}(0.6,0.2)>c_{0}^{A}$, we therefore conclude that the process is capable. We also see that if we ignore the measurements errors, the critical value may be calculated as $c_{0}=1.500$. So if another sample gives $\bar{g}=1592.000$ and $s_{G}=107.694$, we always have $\hat{\xi}_{G}=0.854$, thus $c_{0}^{A}=1.456$ and $c_{0}=1.500$. Since $\hat{c}_{p}^{\prime \prime} G(0.6,0.2)=1.479$, we conclude that the process is capable, but we cannot draw the same conclusion if we ignore the measurement errors.

\section{Conclusion}

In this work we have been concerned with the problem of gauge measurement errors when dealing with process capability indices for one-sided tolerances. Grau [6] introduces the $C_{p}^{u}(u, v)$ and $C_{p}^{l}(u, v)$ indices to cover the processes with one-sided tolerances. The quality of a process is defined by the centring process and the proportion of non-conforming manufactured items. Grau [7] gives the elements that will allow the user to choose the pair $(u, v)$ that will meet his needs as best as possible. In this paper we show that gauge measurement errors have a significant impact on the estimation of a process capability. The estimator evaluating the capability by using the sample data contaminated with gauge measurement errors severely underestimates the $\alpha$-risk, resulting in a smaller testing power. We suggest an adjusted critical value to improve the accuracy of the capability assessment. For practical purpose we give a Maple program helpful to the users for their factory applications. Finally a real example in a company producing polymer granulates is used to assess the interest of the approach.

\section{Appendix A:}

- We have

$$
\begin{aligned}
& \xi^{u}=\max (\xi,-\xi / k), \quad \xi^{l}=\max (\xi / k,-\xi) \\
& \xi=\left\{\begin{array}{l}
\xi^{u} \text { if } \xi>0 \\
-k \xi^{u} \text { if } \xi<0
\end{array}, \quad \xi= \begin{cases}k \xi^{l} & \text { if } \xi>0 \\
-\xi^{l} & \text { if } \xi<0\end{cases} \right. \\
& \xi_{G}^{u}=\max \left(\xi_{G},-\xi_{G} / k\right), \quad \xi_{G}^{l}=\max \left(\xi_{G} / k,-\xi_{G}\right) \\
& \xi_{G}=\left\{\begin{array}{l}
\xi_{G}^{u} \text { if } \xi_{G}>0 \\
-k \xi_{G}^{u} \text { if } \xi_{G}<0
\end{array}, \quad \xi_{G}=\left\{\begin{array}{lll}
k \xi_{G}^{l} & \text { if } & \xi_{G}>0 \\
-\xi_{G}^{l} & \text { if } & \xi_{G}<0
\end{array}\right.\right.
\end{aligned}
$$

- Since $\xi=(\mu-T) / \sigma$ and $\xi_{G}=(\mu-T) / \sigma_{G}$, we have

$$
\begin{aligned}
\xi_{G} & =\xi / \sqrt{1+\tau^{2}} \\
\xi & =\xi_{G} \sqrt{1+\tau^{2}}
\end{aligned}
$$

- From (A.1) and (A.6), we have

$$
\begin{aligned}
& \xi^{u}=\max \left(\xi_{G} \sqrt{1+\tau^{2}},-\xi_{G} \sqrt{1+\tau^{2}} / k\right), \\
& \xi^{l}=\max \left(\xi_{G} \sqrt{1+\tau^{2}} / k,-\xi_{G} \sqrt{1+\tau^{2}}\right)
\end{aligned}
$$

- From (A.3), (A.5) and (A.1), we have

$$
\xi_{G}^{u}=\xi^{u} / \sqrt{1+\tau^{2}}, \quad \xi_{G}^{l}=\xi^{l} / \sqrt{1+\tau^{2}}
$$

- Since $\xi^{u}=A_{u}^{*} / \sigma$ and $\xi^{l}=A_{l}^{*} / \sigma$, from (1) we have 


$$
\begin{gathered}
\xi^{u}=\frac{-C_{p}^{u} u / 3+\sqrt{\left(C_{p}^{u}\right)^{2}(u / 3)^{2}+\left(\left(C_{p}^{u}\right)^{2}-\left(C_{p}^{u}(u, v)\right)^{2}\right)\left(v\left(C_{p}^{u}(u, v)\right)^{2}-(u / 3)^{2}\right)}}{v\left(C_{p}^{u}(u, v)\right)^{2}-(u / 3)^{2}}, \\
\xi^{l}=\frac{-C_{p}^{l} u / 3+\sqrt{\left(C_{p}^{l}\right)^{2}(u / 3)^{2}+\left(\left(C_{p}^{l}\right)^{2}-\left(C_{p}^{l}(u, v)\right)^{2}\right)\left(v\left(C_{p}^{l}(u, v)\right)^{2}-(u / 3)^{2}\right)}}{v\left(C_{p}^{l}(u, v)\right)^{2}-(u / 3)^{2}}
\end{gathered}
$$

$$
\begin{aligned}
C_{p}^{u} & =\sqrt{1+v \xi^{u^{2}}} C_{p}^{u}(u, v)+u \xi^{u} / 3, \\
C_{p}^{l} & =\sqrt{1+v \xi^{2}} C_{p}^{l}(u, v)+u \xi^{l} / 3
\end{aligned}
$$

- In the same manner, from (2) we have

$$
\begin{aligned}
C_{p}^{u G} & =\sqrt{1+v \xi_{G}^{u^{2}}} C_{p}^{u G}(u, v)+u \xi_{G}^{u} / 3, \\
C_{p}^{l G} & =\sqrt{1+v \xi_{G}^{l^{2}}} C_{p}^{l G}(u, v)+u \xi_{G}^{l} / 3
\end{aligned}
$$

- From (A.10) and (3) we have

$$
\begin{aligned}
& C_{p}^{u G}=\frac{\sqrt{1+v \xi_{G}^{u^{2}}} \sqrt{1+v \xi^{u^{2}}}}{\sqrt{1+\tau^{2}+v \xi^{u^{2}}}} C_{p}^{u}(u, v)+u \xi_{G}^{u} / 3, \\
& C_{p}^{l G}=\frac{\sqrt{1+v \xi_{G}^{l^{2}}} \sqrt{1+v \xi^{l^{2}}}}{3 \sqrt{1+\tau^{2}+v \xi^{2}}} C_{p}^{l}(u, v)+u \xi_{G}^{l} / 3
\end{aligned}
$$

- Since $C_{p}^{u G}=C_{p}^{u G}(0,0)$ and $C_{p}^{u}=C_{p}^{u}(0,0)$, from $(3)$

$$
\begin{gathered}
C_{p}^{u G}=C_{p}^{u} / \sqrt{1+\tau^{2}}, \\
C_{p}^{l G}=C_{p}^{l} / \sqrt{1+\tau^{2}}
\end{gathered}
$$

- From (A.12) and (A.11), we have

$$
\begin{aligned}
& C_{p}^{u}=\sqrt{1+\tau^{2}}\left(\frac{\sqrt{1+v \xi_{G}^{u^{2}}} \sqrt{1+v \xi^{u^{2}}}}{\sqrt{1+\tau^{2}+v \xi^{u^{2}}}} C_{p}^{u}(u, v)+u \xi_{G}^{u} / 3\right), \\
& C_{p}^{l}=\sqrt{1+\tau^{2}}\left(\frac{\sqrt{1+v \xi_{G}^{l^{2}}} \sqrt{1+v \xi^{l^{2}}}}{\sqrt{1+\tau^{2}+v \xi^{l^{2}}}} C_{p}^{l}(u, v)+u \xi_{G}^{l} / 3\right)
\end{aligned}
$$

- From (1), by a proof identical to Grau [15], we obtain

$$
\text { see equation (A.14) above }
$$

\section{Appendix B:}

The Maple Program to compute the critical value $c_{0}^{A}$

When the function $H_{G}$ takes values that are too low, the Maple software is sometimes unable to perform the calculation of the integral of $H_{G}$. This is the reason why we limit the domain of integration $\left[0 ; K_{G}\right]$ to the domain $[K 0 ; K 1]$ on which $H_{G}>10^{-8}$. This does not change the value of the integral for the required precision.
Algorithm

Step 1. Read $u, v, k, \tau, n, \alpha, c, \hat{\xi}_{G}, B$ (the tolerance limit)

Step 2. Compute $\hat{\xi}^{u}$ or $\hat{\xi}^{l}$ from (A.7), $\hat{\xi}_{G}^{u}$ or $\hat{\xi}_{G}^{l}$ from (A.3), $C_{p}^{u}$ or $C_{p}^{l}$ from (7) or $(8), C_{p}^{u G}$ or $C_{p}^{l G}$ from (A.12).

Step 3. Compute $H_{G}, K_{G}$.

Step 4. Find the adjusted critical value $c_{0}^{A}$ from (6).

Step 4.1. Find $K 0$ a lower integration bound.

Step 4.2. Find $K 1$ an upper integration bound.

Step 5. Print the adjusted critical value.

Step 6. Plot the power test.

\section{Maple program}

restart:with(stats):

\#1. Read u, v, k (risk),tau (error contamination), n (number of observations), alpha (the alpha-risk), c (threshold value for a capable process), $\mathrm{xG}$ (estimation of (muG$\mathrm{T})$ /sigmaG), B (the bound, L for Lower, U for Upper) $\mathrm{u}:=0.6: \mathrm{v}:=0.2: \mathrm{k}:=3: \mathrm{tau}:=0 . \mathrm{n}:=112$ alpha: $=0.05: \mathrm{c}:=1.33$ : $\mathrm{xG}:=0.854: \mathrm{B}:=\mathrm{L}$ :

\#2. Compute xB, xBG, CpB, CpBG

if $(\mathrm{B}=\mathrm{L})$ then $\mathrm{xB}:=\max \left(\mathrm{xG}^{*} \operatorname{sqrt}\left(1+\operatorname{tau}^{\wedge} 2\right) / \mathrm{k},-\right.$ $\left.\mathrm{xG}^{*} \operatorname{sqrt}\left(1+\tan ^{\wedge} 2\right)\right)$ else $\mathrm{xB}:=\max \left(\mathrm{xG}^{*} \operatorname{sqrt}\left(1+\operatorname{tau}^{\wedge} 2\right)\right.$,$\left.\mathrm{xG}^{*} \operatorname{sqrt}\left(1+\operatorname{tau}^{\wedge} 2\right) / \mathrm{k}\right)$ fi:

if $(B=L)$ then $x B G:=\max (x G / k,-x G)$ else $\mathrm{xBG}:=\max (\mathrm{xG},-\mathrm{xG} / \mathrm{k})$ fi:

$\mathrm{CpB}:=\operatorname{sqrt}\left(1+\operatorname{tau}^{\wedge} 2\right)^{*}\left(\operatorname{sqrt}\left(1+\mathrm{v}^{*} \mathrm{xBG}^{\wedge} 2\right)^{*} \operatorname{sqrt}\left(1+\mathrm{v}^{*} \mathrm{xB}^{\wedge} 2\right)\right.$ $\left./ \operatorname{sqrt}\left(1+\operatorname{tau}^{\wedge} 2+\mathrm{v}^{*} \times \mathrm{B}^{\wedge} 2\right)^{*} \mathrm{c}+\mathrm{u}^{*} \mathrm{xBG} / 3\right)$ :

$\mathrm{CpBG}:=\mathrm{CpB} / \operatorname{sqrt}\left(1+\operatorname{tau}^{\wedge} 2\right)$ :

\#3. Compute $\mathrm{H}$ and $\mathrm{K}$

$\mathrm{F}:=(\mathrm{c} 0, \mathrm{t})->$ stats $[$ statevalf,cdf,chisquare $[\mathrm{n}-$

1]] $\left(\left(3^{*} \mathrm{CpBG}^{*} \operatorname{sqrt}(\mathrm{n})-\mathrm{u}^{*} \mathrm{t}\right)^{\wedge} 2 / 9 / \mathrm{c}^{\wedge} 2-\mathrm{v}^{*} \mathrm{t}^{\wedge} 2\right)$ :

if $(\mathrm{B}=\mathrm{L})$

then $\mathrm{fB}:=\mathrm{t}->\mathrm{k}^{*}$ stats [statevalf,pdf,normald] $\left(\mathrm{k}^{*} \mathrm{t}-\mathrm{x} \mathrm{G}^{*} \mathrm{n}^{\wedge} .5\right)$

+ stats[statevalf,pdf,normald] $\left(\mathrm{t}+\mathrm{xG}^{*} \mathrm{n}^{\wedge} .5\right)$

else $\mathrm{fB}:=\mathrm{t}->$ stats[statevalf,pdf,normald] $\left(\mathrm{t}-\mathrm{xG} \mathrm{K}^{*} \mathrm{n}^{\wedge} .5\right)$

$+\mathrm{k}^{*}$ stats[statevalf,pdf,normald] $\left(\mathrm{k}^{*} \mathrm{t}+\mathrm{xG}^{*} \mathrm{n}^{\wedge} .5\right)$

fi:

$\mathrm{H}:=(\mathrm{c} 0, \mathrm{t})->\mathrm{F}(\mathrm{c} 0, \mathrm{t}) * \mathrm{fB}(\mathrm{t}):$

$\mathrm{Kl}:=3^{*} \mathrm{CpBG}^{*} \operatorname{sqrt}(\mathrm{n}) /\left(\mathrm{u}+3^{*} \mathrm{c} 0 * \operatorname{sqrt}(\mathrm{v})\right)$ :

\#4. Compute $\mathrm{c} 0$ the critical value

$\mathrm{k} 1:=0: \mathrm{k} 3:=5.9$ :

for i from 1 to 100

do $\mathrm{k} 2:=(\mathrm{k} 1+\mathrm{k} 3) / 2: \mathrm{c} 0:=\operatorname{evalf}(\mathrm{k} 2)$ :

\# 4.1.Find K0, lower integration bound

$\mathrm{K} 0:=0: \mathrm{z} 1:=\operatorname{evalf}(\mathrm{H}(\mathrm{c} 0, \mathrm{~K} 0))$ : 
if $\mathrm{z} 1<1 \mathrm{e}-8$ then for $\mathrm{j}$ from 1 to $\operatorname{trunc}(\mathrm{Kl})$

do $\mathrm{z} 1:=\operatorname{evalf}(\mathrm{H}(\mathrm{c} 0, \mathrm{j}))$;

if $\mathrm{z} 1>1 \mathrm{e}-8$ then for $\mathrm{h}$ from $\mathrm{j}-0.9$ by 0.1 to $\mathrm{j}$

do $\mathrm{z} 1:=\operatorname{evalf}(\mathrm{H}(\mathrm{c} 0, \mathrm{~h}))$;

if $\mathrm{z} 1>1 \mathrm{e}-8$ then $\mathrm{K} 0:=\mathrm{h}-0.1$ :break fi:

od:

break fi:

od:

fi:

\# 4.2.Find K1, upper integration bound

$\mathrm{K} 1:=\operatorname{evalf}(\mathrm{Kl})-0.00001: \mathrm{z} 2:=\operatorname{evalf}(\mathrm{H}(\mathrm{c} 0, \mathrm{~K} 1))$ :

if $\mathrm{z} 2<1 \mathrm{e}-8$ then for $\mathrm{j}$ from $\mathrm{K} 1$ by -1 to 0

do $\mathrm{z} 2:=\operatorname{evalf}(\mathrm{H}(\mathrm{c} 0, \mathrm{j}))$;

if $z 2>1 e-8$ then for $h$ from $j+0.9$ by -0.1 to $j$

do $\mathrm{z} 2:=\operatorname{evalf}(\mathrm{H}(\mathrm{c} 0, \mathrm{~h}))$;

if $\mathrm{z} 2>1 \mathrm{e}-8$ then $\mathrm{K} 1:=\mathrm{h}+0.1$ :break fi:

od:

break fi:

od:

fi:

if $\mathrm{z} 1<1 \mathrm{e}-8$ and $\mathrm{z} 2<1 \mathrm{e}-8$ then $\mathrm{y}:=0$

else $\mathrm{y}:=\operatorname{evalf}(\operatorname{int}(\mathrm{H}(\mathrm{c} 0, \mathrm{t}), \mathrm{t}=\operatorname{evalf}(\mathrm{K} 0) .$. evalf $((\mathrm{K} 0+\mathrm{K} 1) / 2)))$

$+\operatorname{evalf}(\operatorname{int}(\mathrm{H}(\mathrm{c} 0, \mathrm{t}), \mathrm{t}=\operatorname{evalf}((\mathrm{K} 0+\mathrm{K} 1) / 2)$..evalf $(\mathrm{K} 1)))$ : fi:

if $\mathrm{y}<$ alpha then $\mathrm{k} 3:=\mathrm{k} 2$ else $\mathrm{k} 1:=\mathrm{k} 2 \mathrm{fi}$ :

if $\operatorname{abs}(y$-alpha $)<0.00001$ then break fi:

od:

\#5. Result

printf("the critical value is $\% .3 \mathrm{f}$ with $\% .2 \mathrm{f}$ per cent

risk",c0,100*alpha);

\#6. Plot the power test

$\mathrm{xB} 1:=\left(-\mathrm{CpB}^{*} \mathrm{u} / 3+\operatorname{sqrt}\left(\mathrm{CpB}^{\wedge} 2^{*}(\mathrm{u} / 3)^{\wedge} 2+\left(\mathrm{CpB}^{\wedge} 2-\right.\right.\right.$

$\left.\left.\left.\mathrm{CpBuv}^{\wedge} 2\right)^{*}\left(\mathrm{v}^{*} \mathrm{CpBuv}^{\wedge} 2-(\mathrm{u} / 3)^{\wedge} 2\right)\right)\right) /\left(\mathrm{v}^{*} \mathrm{CpBuv}^{\wedge} 2-\right.$

$\left.(\mathrm{u} / 3)^{\wedge} 2\right)$ :

$\mathrm{xBG} 1:=\mathrm{xB} 1 / \operatorname{sqrt}\left(1+\operatorname{tau}^{\wedge} 2\right)$ :

if $(\mathrm{B}=\mathrm{L})$ then $\mathrm{xG} 1:=$ piecewise $\left(\mathrm{xG}>0, \mathrm{k}^{*} \mathrm{xBG} 1,-\mathrm{xBG} 1\right)$

else $x \mathrm{G} 1:=$ piecewise $\left(x G>0, x B G 1,-k^{*} x B G 1\right)$ fi:

if $(\mathrm{B}=\mathrm{L})$ then $\mathrm{fB}:=\mathrm{t}->\mathrm{k}^{*}$ stats [statevalf,pdf,normald] $(\mathrm{k} * \mathrm{t}-$

$\left.\mathrm{xG} 1{ }^{*} \mathrm{n}^{\wedge} .5\right)+$ stats[statevalf,pdf,normald] $\left(\mathrm{t}+\mathrm{xG} 1^{*} \mathrm{n}^{\wedge} .5\right)$

else $\quad \mathrm{fB}:=\mathrm{t}$-stats[statevalf,pdf,normald] $\left(\mathrm{t}-\mathrm{xG} 1{ }^{*}{ }^{\wedge}{ }^{\wedge} .5\right)$

$+\mathrm{k}^{*}$ stats[statevalf,pdf,normald] $\left(\mathrm{k} * \mathrm{t}+\mathrm{xG} 1^{*} \mathrm{n}^{\wedge} .5\right)$ fi:

$\mathrm{pw}:=\mathrm{c} 0->\operatorname{int}(\mathrm{F}(\mathrm{c} 0, \mathrm{t}) * \mathrm{fB}(\mathrm{t}), \mathrm{t}=0 . . \mathrm{K} 1)$ :

$\operatorname{plot}(\mathrm{pw}(\mathrm{c} 0), \mathrm{CpBuv}=\mathrm{c} . \mathrm{CpB}, \mathrm{title}=$ "test power" $)$;

\section{References}

1. L.K. Chan, S.W. Cheng, F.A. Spiring, A New Measure of Process Capability: $\mathrm{C}_{\mathrm{pm}}$, J. Qual. Technol. 20, 162-175 (1998)

2. W.L. Pearn, S. Kotz, N.L. Johnson, Distributional and inferential properties of process capability indices, J. Qual. Technol. 24, 216-231 (1992)

3. K. Vännman, A unified approach to capability indices, Stat. Sin. 5, 805-820 (1995)

4. K.S. Chen, W.L. Pearn, Capability indices for processes with asymmetric tolerances, J. Chin. Inst. Eng. 24, 559 $568(2001)$

5. D. Grau, Process yield and capability indices, Commun. Stat. Theory Meth. 40, 2751-2771 (2011)

6. D. Grau, New Process capability indices for one-sided tolerances, Qual. Technol. Quant. Manag. 6, 107-124 (2009)

7. D. Grau, Process yield, process centering, and capability indices for one-sided tolerance processes, Qual. Technol. Quant. Manag. 9, 153-170 (2012)

8. H.J. Mittag, Measurement error effects on the performance of process capability indices, Front. Stat. Qual. Control 5, 195-206 (1997)

9. W.L. Pearn, M.Y. Liao, Estimating and testing process precision with presence of gauge measurement errors, Qual. Quant. 41, 757-777 (2007)

10. W.L. Pearn, M.Y. Liao, Measuring process capability based on $\mathrm{C}_{\mathrm{pk}}$ with gauge measurement errors, Microelectron. Reliab. 45, 739-751 (2005)

11. W.L. Pearn, M.Y. Liao, One-sided process capability assessment in the presence of measurement errors, Qual. Rel. Eng. Int. 22, 771-785 (2006)

12. W.L. Pearn, M.H. Shu, B.M. Hsu, Testing process capability based on $\mathrm{C}_{\mathrm{pm}}$ in the presence of random measurement errors, J. Appl. Stat. 32, 1003-1024 (2005)

13. B.M. Hsu, M.H. Shu, W.L. Pearn, Measuring process capability based on $\mathrm{C}_{\mathrm{pmk}}$ with gauge measurement errors, Qual. Rel. Eng. Int. 23, 597-614 (2007)

14. D. Grau, Lower confidence bound for capability indices with asymmetric tolerances and gauge measurement errors, Int. J. Qual. Eng. Technol. 2, 212-228 (2011)

15. D. Grau, Testing capability indices for manufacturing processes with asymmetric tolerance limits and measurement errors, Int. J. Metrol. Qual. Eng. 2, 61-73 (2011) 